Supporting Information

\title{
Laminated Graphene Films for Flexible Transparent Thin
}

\section{Film Encapsulation}

Hong-Kyu Seo, Min-Ho Park, Young-Hoon Kim, Sung-Joo Kwon, Su-Hun Jeong and TaeWoo Lee*

Department of Materials Science and Engineering, Pohang University of Science and Technology (POSTECH), Pohang, Gyungbuk 790-784, Republic of Korea.

*E-mail: twlee@ postech.ac.kr, taewlees@gmail.com 




Figure S1. Schematic of Ca oxidation test sample. Ca films $(200 \mathrm{~nm})$ with an area of $1.2 \times$ $1.2 \mathrm{~cm}$ were deposited on patterned $\mathrm{Al}$ electrodes $(100 \mathrm{~nm})$ on glass substrates.



Figure S2. The WVTR values (left y axis) and lag times (right y axis) vs. several encapsulants. 
(a)

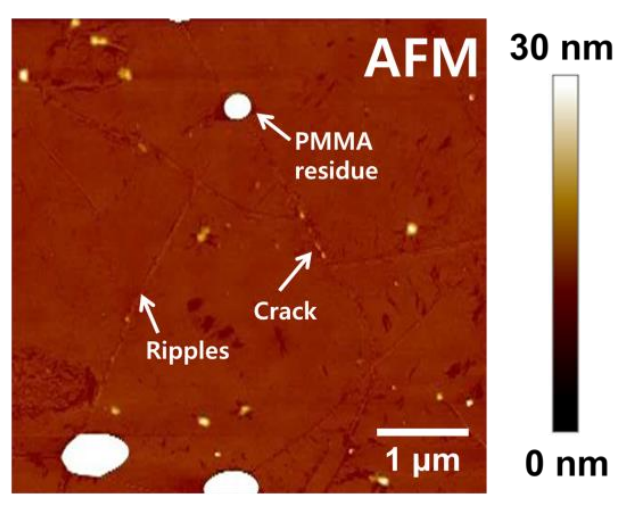

(b)

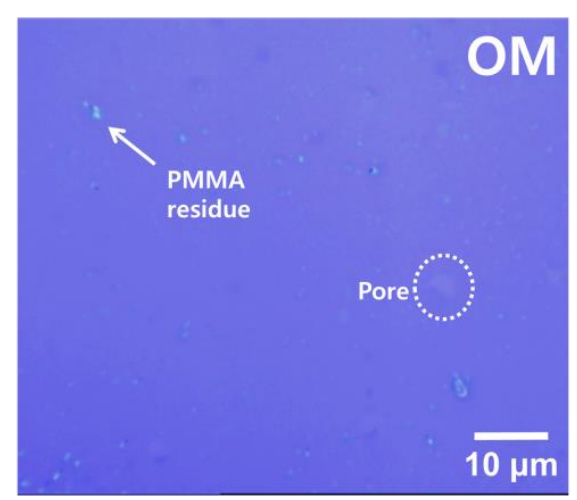

Figure S3. (a) Atomic force microscopy (AFM) and (b) optical microscope images of singlelayer graphene surface that has several defects. 